\title{
钛酸铋钠基无铅铁电陶瓷场致应变性能研究
}

\author{
杨慧平 ${ }^{1}$ ，周学凡 ${ }^{1}$, 方豪杰 ${ }^{2}$, 张晓云 ${ }^{2}$, 罗 行 $^{1}$, 张 ${ }^{1}$ \\ (1. 中南大学 粉末冶金研究院, 粉末冶金国家重点实验室, 长沙 410083; 2. 湖南省美程陶瓷科技有限公司, 娄底 417000)
}

摘 要: 钛酸铋钠 $\left(\mathrm{Bi}_{0.5} \mathrm{Na}_{0.5}\right) \mathrm{TiO}_{3}(\mathrm{BNT})$ 作为典型的钻钛矿型弛豫铁电体, 具有超高的场致应变, 是最有希望代替铅 基体系的无铅压电体系之一。与铅基陶瓷相比, BNT 基陶瓷具有驱动电压较高、迟滞较大以及温度稳定性差等劣势。 为了优化无铅驱动器的应变性能, 本研究采用固相反应法制备 $(1-x)\left\{0.76\left(\mathrm{Bi}_{0.5} \mathrm{Na}_{0.5}\right) \mathrm{TiO}_{3}-0.24 \mathrm{SrTiO}_{3}\right\}-x \mathrm{NaNbO}_{3}\left(\mathrm{BNT}^{-}\right.$ ST- $x$ NN, $x=0 \sim 0.03)$ 无铅铁电陶瓷。结果表明, 当 $x=0.01$ 时, 该陶瓷在较低电场 $(E=4 \mathrm{kV} / \mathrm{mm})$ 下的应变值可达到 $0.278 \%$, 等 效压电系数 $d_{33}^{*}$ 高达 $695 \mathrm{pm} / \mathrm{V}$ 。此时, 陶瓷处于非遍历/遍历弛豫相界处, 电场诱导弛豫一铁电相变导致大场致应变。 与 $x=0.01$ 相比, $x=0.02$ 时应变值为 $0.249 \%$, 略微下降, 但迟滞却降低至 $43 \%$ 。此外, 该应变在 $25 \sim 100{ }^{\circ} \mathrm{C}$ 温度范围 内维持稳定。本研究表明, 在 $\mathrm{BNT}$ 基陶瓷中固溶 $\mathrm{SrTiO}_{3}$ 和 $\mathrm{NaNbO}_{3}$ 组元可以提高场致应变值, 同时维持较低的驱 动电场和良好的温度稳定性, 可用于压电驱动器研制。

关 键 词: 钛酸铋钠; 无铅铁电陶瓷; 场致应变; 弛豫态

中图分类号: TQ174 文献标志码: A

\section{Field-induced Strain Property of Lead-free Ferroelectric Ceramics Based on Sodium Bismuth Titanate}

YANG Huiping ${ }^{1}$, ZHOU Xuefan ${ }^{1}$, FANG Haojie ${ }^{2}$, ZHANG Xiaoyun $^{2}$, LUO Hang ${ }^{1}$, ZHANG Dou ${ }^{1}$

(1. State Key Laboratory of Powder Metallurgy, Powder Metallurgy Research Institute, Central South University, Changsha 410083, China; 2. Hunan Meicheng Ceramic Technology Co., Ltd., Loudi 417000, China)

Abstract: Piezoelectric actuators have advantages of fast response, high positioning accuracy, small size, and have received widespread attention in the field of precision drives. Lead-based piezoelectric actuators occupy the main commercial market. To avoid the use of the harmful element lead, lead-free piezoelectric materials and actuators must be developed. Among them, bismuth sodium titanate $\left(\mathrm{Bi}_{0.5} \mathrm{Na}_{0.5}\right) \mathrm{TiO}_{3}(\mathrm{BNT})$, was reported but it has some disadvantages of higher driving voltage, larger hysteresis, and poor temperature stability. To optimize the strain performance of the lead-free actuator, this study adopted the solid-phase reaction method to prepare $(1-\chi)\left\{0.76\left(\mathrm{Bi}_{0.5} \mathrm{Na}_{0.5}\right) \mathrm{TiO}_{3^{-}}\right.$ $\left.0.24 \mathrm{SrTiO}_{3}\right\}-x \mathrm{NaNbO}_{3}(\mathrm{BNT}-\mathrm{ST}-x \mathrm{NN}, x=0-0.03)$ lead-free ferroelectric ceramics and systematically studied its fieldinduced strain performance. The results show that when $x=0.01$, the strain value of the ceramic can reach $0.278 \%$ under a low electric field $(E=4 \mathrm{kV} / \mathrm{mm})$, and the piezoelectric coefficient $d_{33}^{*}$ is as high as $695 \mathrm{pm} / \mathrm{V}$. Meanwhile,

收稿日期：2021-07-16; 收到修改稿日期：2021-09-28; 网络出版日期：2021-11-01

基金项目: 国家重点研发项目(2020YFA0711700); 国家自然科学基金(U19A2087); 湖南省创新型省份建设专项(2020GK2062); 博士后创新人才支持计划(BX2021377)

National Key Research and Development Project of China (2020YFA0711700); National Natural Science Foundation of China (U19A2087); Special Funding Support for the Construction of Innovative Provinces in Hunan Province of China (2020GK2062); China National Postdoctoral Program for Innovative Talents (BX2021377)

作者简介: 杨慧平(1994-)，女，硕士研究生. E-mail: 3150289484@qq.com YANG Huiping (1994-), female, Master candidate. E-mail: 3150289484@qq.com

通信作者：张 斗，教授. E-mail: dzhang@csu.edu.cn ZHANG Dou, professor. E-mail: dzhang@csu.edu.cn 
the ceramic is at the non-ergodic/ergodic relaxation phase boundary, and the electric field induced relaxor-ferroelectric phase transition leads to large field-induced strain. Compared with $x=0.01$, the strain value at $x=0.02$ is $0.249 \%$, which is slightly reduced, but the hysteresis is significantly reduced to $43 \%$ of the comparator. In addition, the strain remains stable in the temperature range of $25-100{ }^{\circ} \mathrm{C}$. This study shows that introduction of $\mathrm{SrTiO}_{3}$ and $\mathrm{NaNbO}_{3}$ into BNT can increase the field-induced strain value while maintaining a low driving voltage and good temperature stability, indicating suitability for the development of piezoelectric actuators.

Key words: sodium bismuth titanate; lead-free ferroelectric ceramics; field-induced strain; relaxed state

作为一种实现电能和机械能相互转化的功能材 料, 压电材料每年有近百亿元的市场, 主要用作驱动、 传感和储能等电子器件 ${ }^{[1]}$ 。其中, 压电驱动器可用作 超声波清洗机、汽车喷油器以及镜头用超声波电机 等, 具有广阔的市场前景, 已成为当前研究热点 ${ }^{[1-2]}$ 。 目前含铅压电驱动器材料锆铁酸铅 $\mathrm{Pb}\left(\mathrm{Zr}_{1-\chi} \mathrm{Ti}_{x}\right) \mathrm{O}_{3}(\mathrm{PZT})$ 在商业应用中占据主要市场, 但由于铅元素对人体 健康和环境都具有极大危害, 已被多国限制使用。 因此，函需开发高性能、环境友好的无铅铁电陶瓷 材料以取代传统铅基压电陶瓷材料。目前, 关于无 铅压电材料和应变性能研究主要集中于三类铁电材 料: 钣酸铋钠 $\left(\mathrm{Bi}_{0.5} \mathrm{Na}_{0.5}\right) \mathrm{TiO}_{3}(\mathrm{BNT})$ 、钛酸钡 $\mathrm{BaTiO}_{3}$ (BT) 和铌酸钾钠 $\left(\mathrm{K}_{0.5} \mathrm{Na}_{0.5}\right) \mathrm{NbO}_{3}(\mathrm{KNN})^{[3]}$ 。优化工艺 与晶粒尺寸、陶瓷织构化以及通过化学掺杂构造准 同型相界(MPB)等是提高陶瓷场致应变性能的有效 方式 ${ }^{[4]}$ 。

通常, $\mathrm{KNN}$ 基铁电陶瓷的压电系数 $d_{33}$ 仅有 $80 \mathrm{pC} / \mathrm{N}$, 通过优化工艺可达 $125 \mathrm{pC} / \mathrm{N}$ 左右, 进一 步调控 $\mathrm{K}$ 和 $\mathrm{Na}$ 的化学计量比, 即 $\mathrm{K} / \mathrm{Na}$ 为 $48 / 52$ 时, $d_{33}$ 可达 $160 \mathrm{pC} / \mathrm{N}^{[5]}$ 。其次, 通过化学掺杂构造 $\mathrm{KNN}$ 基陶瓷的 MPB, 也能提升陶瓷的压电性能。2018 年 LV 等 ${ }^{[6]}$ 通过掺杂构造 $(0.99-x) \mathrm{KNNS}-0.01 \mathrm{SZ}-x \mathrm{BNZ}$ 陶瓷, 使其在 MPB 处压电系数 $d_{33}$ 可达 $450 \mathrm{pC} / \mathrm{N}$ 且应变值在 $0.148 \% \sim 0.16 \%$ 范围内。此外, 多晶体陶 瓷织构化也能显著优化陶瓷应变性能 ${ }^{[7]}$ 。2004 年, Saito 等 ${ }^{[8]}$ 通过掺杂 $\mathrm{Li} 、 \mathrm{Ta}$ 和 $\mathrm{Sb}$ 等元素, 在 MPB 处 得到 $<001>$ 方向织构的 KNN 基铁电陶瓷，该陶瓷的 $d_{33}$ 高达 $416 \mathrm{pC} / \mathrm{N}$ 且 $d_{33}^{*}$ 为 $750 \mathrm{pm} / \mathrm{V}$ 。然而, 由于 $\mathrm{K}$ 元素极易挥发, 普通烧结很难得到致密陶瓷, 极大 地阻碍了 $\mathrm{KNN}$ 基铁电陶瓷的实际应用 ${ }^{[9]}$ 。 $\mathrm{BT}$ 是最 早发现的无铅铁电材料, 优化应变性能方法与 KNN 类似 ${ }^{[10]}$ 。2018 年 Zhao 等 ${ }^{[11]}$ 构造 $(1-x-y) \mathrm{BaTiO}_{3}$ $y \mathrm{CaTiO}_{3}-x\left(\mathrm{BaZr}_{1-z} \mathrm{Hf}_{z}\right) \mathrm{O}_{3}(\mathrm{BT}-y \mathrm{CT}-x \mathrm{BZHz})$ 陶瓷, 在 MPB 处获得堪比铅基陶瓷的压电性能: $d_{33}=500 \mathrm{pC} / \mathrm{N}$ 与 $d_{33}^{*}=1100 \mathrm{pm} / \mathrm{V}$ 。再者, 优化晶粒尺寸和取向也是 有效途径。Tan 等 ${ }^{[12]}$ 对 BT 陶瓷晶粒尺寸与应变关
系研究发现, 在晶粒尺寸为 $4.5 \mu \mathrm{m}$ 时, 场致应变最 佳。此外, Zhao 等 ${ }^{[13]}$ 发现, BT 作为籽晶的 BZT-BCT 织构陶瓷, 可在极低电场 $E=20 \mathrm{kV} / \mathrm{cm}$ 时, 得到 $0.153 \%$ 的应变值, $d_{33}^{*}$ 可达 $767 \mathrm{pm} / \mathrm{V}$ 。即便如此, 目 前 $\mathrm{BT}$ 基陶瓷场致应变仍然较低 $(0.1 \% \sim 0.2 \%)$, 且居 里温度只有 $120{ }^{\circ} \mathrm{C}$, 用作压电驱动器件并不具有显 著优势。2007 年, 张善涛课题组 ${ }^{[14]}$ 发现 0.92BNT$0.06 \mathrm{BT}-0.02 \mathrm{KNN}$ 陶瓷具有与商用 PZT 陶瓷相媲美 的应变性能, 该陶瓷在 $E=80 \mathrm{kV} / \mathrm{cm}$ 的电场下, 应变值 高至 $0.45 \%$ 。该研究发表至今, BNT 基陶瓷的场致 应变和驱动性能优化成为相关领域的研究热点。与 前两类无铅陶瓷不同, BNT 基陶瓷除了常见的三方 一四方准同型相界 MPB(I)以外, 还具有极性铁电相 与非极性弛豫相的准同型相界 MPB(II)。这是由于 $\mathrm{BNT}$ 晶格的 $\mathrm{A}$ 位被 $\mathrm{Bi}$ 与 $\mathrm{Na}$ 两个异价阳离子同时 占据, 造成 A 位化学无序, 铁电畴演变为极性纳米 微区 PNRs, 呈现非极性弛豫相。BNT 基材料的弛 豫态包括“非遍历弛豫态”与 “遍历弛豫态”, 区别在 于外场撤除后铁电相能否稳定存在。遍历弛豫态是 可逆的, 因此也是不稳定的, 而非遍历弛豫态则不 可逆。研究者们发现, 在 MPB(I)处, BNT 基陶瓷的 压电性能 $d_{33}$ 有效提高, 而处于 $\mathrm{MPB}(\mathrm{II})$ 时, 陶瓷的 场致应变性能显著升高 ${ }^{[15]}$ 。通过对 0.92BNT$0.06 \mathrm{BT}-0.02 \mathrm{KNN}$ 陶瓷场致应变原理研究发现, 这 主要源于室温下电场诱导非极性弛豫一极性铁电相 转变 ${ }^{[16]}$ 。基于上述研究, 降低 $\mathrm{MPB}(\mathrm{II})$ 相界温度 $T_{\mathrm{d}}$ 至室温来优化应变性能不失为一种有效思路, 主要 手段包括构造钙钛矿型多组元固溶体、离子掺杂以 及调节非化学计量比等。如 2013 年, Hao 等 ${ }^{[17]}$ 制备 BNT-BKT-KNN 陶瓷, 得到应变值 $S=0.48 \%$, 且 $d_{33}^{*}=600 \mathrm{pm} / \mathrm{V}$ 。Chen 等 ${ }^{[18]}$ 将 $\mathrm{LiNbO}_{3}$ 掺杂到 BNT-6BT 体系得到 $S=0.6 \%$ 和 $d_{33}^{*}=857 \mathrm{pm} / \mathrm{V}$ 。然而, 与商用软 PZT 陶瓷相比, BNT 基陶瓷的驱动电场通常需要 $60 \sim 80 \mathrm{kV} / \mathrm{cm}$, 是 PZT 驱动电场的 2 倍多。同时, 大 场致应变值常伴随着温度使用范围降低与迟滞升高 的弊端。其中，迟滞(Hysteresis, $H$ )的计算公式为: 


$$
H=\frac{\Delta S}{S_{\max }}, \Delta S=S^{\frac{E}{2}}{ }_{\text {max }}-S^{\frac{E}{2}} \text { mini }
$$

式中, $H$ 代表迟滞, $S_{\max }$ 代表在驱动电场 $E$ 下最大的 应变值, $S_{\text {max }}^{E / 2}$ 与 $S^{E / 2}{ }_{\text {mini }}$ 分别代表在二分之一电场 $E$ 下最大与最小的应变值, $\Delta S$ 为上述两者之差。

1974 年, Sakata 等 ${ }^{[17]}$ 首次发现 $(1-x)$ BNT- $x$ ST 陶 瓷在低电场得到大场致应变。随后, Hiruma 等 ${ }^{[19]}$ 对 该陶瓷进行系统研究, 发现这是因为 $\mathrm{ST}$ 具有高度 容纳系数 $t$, 使 $x=0.26 \sim 0.28$ 时 BNT 基材料形成 $\mathrm{MPB}(\mathrm{II})$, 且当 $x=0.28$ 时场致应变值最佳, 达 $0.29 \%$ 。这 是由于 $\mathrm{ST}$ 能够有效地破坏长程有序的铁电相, 并 且将相转变温度 $T_{\mathrm{d}}$ 降低到室温, 从而使其能在室温 下得到低场一场致应变 ${ }^{[19-20]}$ 。此外, 同样为钻钛矿结 构的 NN 也能有效提高 BNT 基陶瓷的应变性能 ${ }^{[21-22]}$ 。 因此, 本研究采用固相反应法制备 BNT-ST- $x$ NN 三 元固溶体陶瓷片, 通过调控 NN 含量, 降低 MPB(II) 相转变温度 $T_{\mathrm{d}}$ 至室温以获得优异的场致应变, 同时 降低驱动电场和应变迟滞。

\section{1 实验方法}

\subsection{BNT-ST- $x$ NN 陶瓷片制备}

采用固相反应合成法制备 $(1-x)\left\{0.76\left(\mathrm{Bi}_{0.5} \mathrm{Na}_{0.5}\right) \mathrm{TiO}_{3}\right.$ $\left.0.24 \mathrm{SrTiO}_{3}\right\}-x \mathrm{NaNbO}_{3}(\mathrm{BNT}-\mathrm{ST}-x \mathrm{NN}, \quad x=0 \sim 0.03)$ 陶 瓷。按照化学计量比分别称取 $\mathrm{Na}_{2} \mathrm{CO}_{3}$ (纯度 $99.8 \%$ )、 $\mathrm{Bi}_{2} \mathrm{O}_{3}$ (纯度 $99 \%$ )、 $\mathrm{SrCO}_{3}$ (纯度 $99 \%$ )、 $\mathrm{TiO}_{2}$ (纯度 $98 \%$ ) 和 $\mathrm{Nb}_{2} \mathrm{O}_{5}$ (纯度 $99.99 \%$ ), 以上药品都为国药集团生 产。将配好的粉末放入装有二氧化锆球的尼龙罐中, 加入乙醇精后装在行星球磨机上, 以 $250 \mathrm{r} / \mathrm{min}$ 转速 球磨 $24 \mathrm{~h}$ 。将球磨后的样品放入烘箱中干燥, 在坩 埚中以 $5{ }^{\circ} \mathrm{C} / \mathrm{min}$ 从室温升温至 $850{ }^{\circ} \mathrm{C}$ 保温 $3 \mathrm{~h}$, 随 炉冷却。将煅烧后的粉末再次以相同条件球磨, 加入 质量分数为 $1 \%$ 的 PVA 黏接剂进行造粒、压片, 并在
$1160{ }^{\circ} \mathrm{C}$ 下保温 $2 \mathrm{~h}$ 烧结成陶瓷片。将烧成的陶瓷片用 砂纸磨成厚度约为 $0.6 \mathrm{~mm}$ 的圆片, 将其双面涂上铂电 极, 并在 $850{ }^{\circ} \mathrm{C}$ 保温 $10 \mathrm{~min}$ 后随炉冷却, 随后可用于 电学性能测试。

\subsection{BNT-ST- $x$ NN 陶瓷片相结构、表面形貌以 及铁电性能表征}

采用 $\mathrm{X}$ 射线衍射分析仪(XRD, D8 Advance, Bruker)检测样品晶相结构。用场发射扫描电子显微 镜(SEM, JEOL-6360LV)对 BNT-ST- $x$ NN 陶瓷的晶粒 尺寸、微观形貌进行表征。材料的铁电和应变性能 采用配备了高精度激光位移传感器的铁电分析仪 (TF Analyzer2000, aixACCT)测试, 介电性能则是通 过介电温谱仪(DMS-500, Partulab)测试得到。

\section{2 结果与讨论}

\subsection{BNT-ST- $x$ NN 陶瓷相结构和微观形貌}

图 1(a)给出了 BNT-ST- $x \mathrm{NN}(x=0 \sim 0.03)$ 陶瓷的 XRD 图谱。结果表明所有组分陶瓷均为典型的钙钛 矿结构, 呈现伪立方相, 没有明显第二相, 此现象 说明 ST 与 NN 都完全固溶进 BNT 陶瓷晶格中, 形 成均匀的三元固溶体陶瓷。图 $1(b, c)$ 分别为陶瓷 XRD 图谱的(111)和(200)衍射峰的放大图像。结果 显示除 $\kappa_{\alpha 2}$ 的峰以外, 没有明显的分裂, 即不存在明 显的三方和四方的晶格畸变。

图 2 为 BNT-ST- $x \mathrm{NN}(x=0 \sim 0.03)$ 陶瓷的表面微观 形貌图。从该 SEM 照片得知所有组分的陶瓷片表面 都无明显缺陷, 结构紧密。再者, 晶粒尺寸呈现不均 匀分布, 大晶粒与小晶粒相互嵌合构成致密结构。 此外, $\mathrm{NN}$ 的加入量与陶瓷晶粒尺寸无明显相关性, 因此可忽略晶粒尺寸对材料宏观电学性能的影响, 可归因于加入 $\mathrm{NN}$ 并不会导致电荷不平衡, 陶瓷中 氧空位含量没有明显变化。
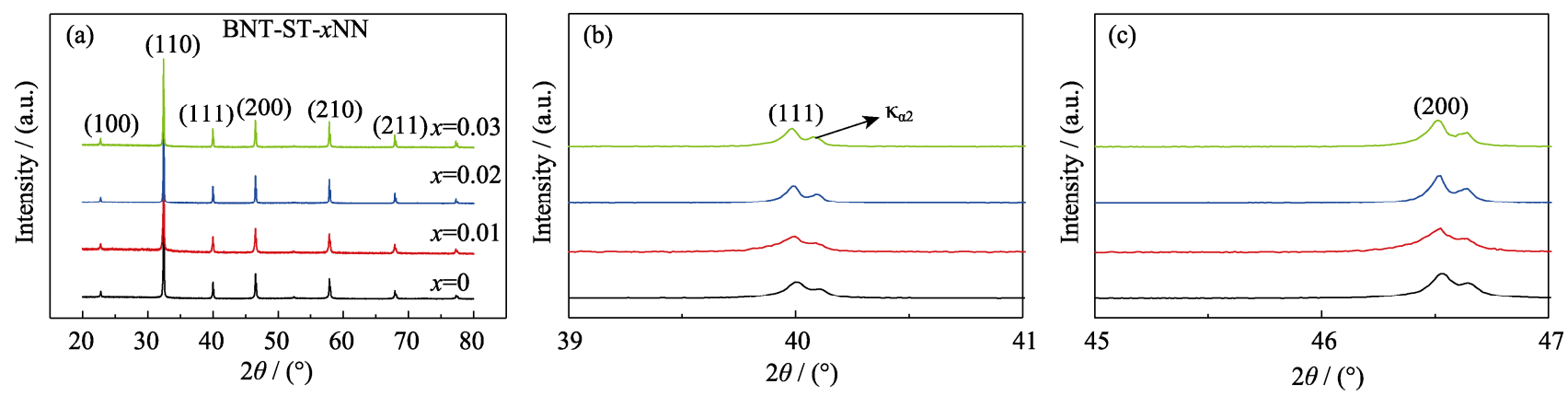

图 1 BNT-ST- $x$ NN 陶瓷的 XRD 图谱和衍射峰放大图

Fig. 1 XRD patterns of BNT-ST- $x$ NN ceramics and diffraction peak magnified patterns (a) XRD patterns of BNT-ST- $x$ NN ceramics; (b) Magnified patterns of $\{111\}$; (c) Magnified patterns of $\{200\}$ 

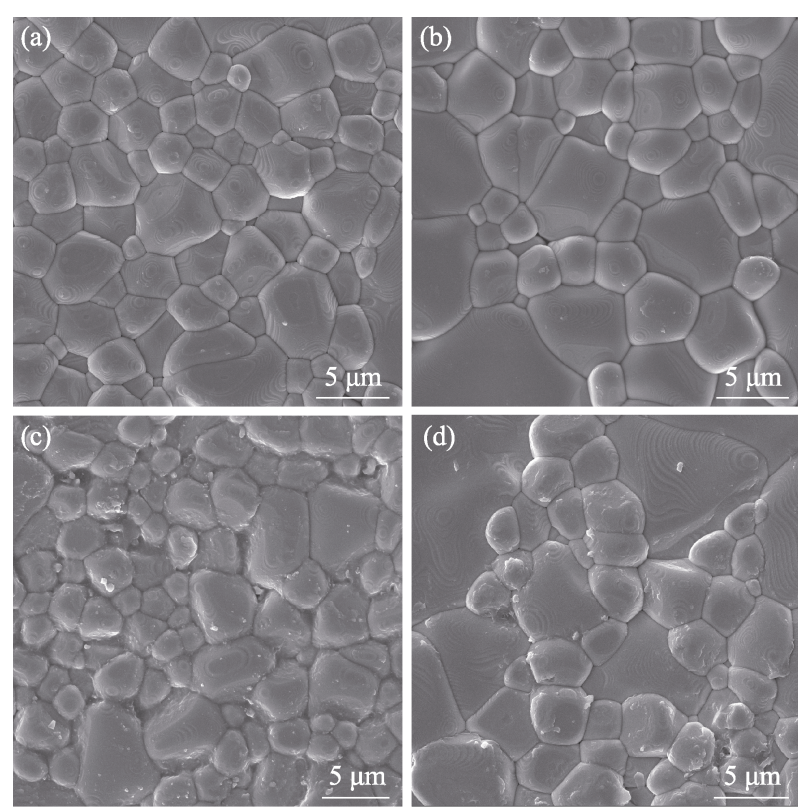

图 2 BNT-ST- $x$ NN 陶瓷的 SEM 照片

Fig. 2 SEM images of BNT-ST- $x$ NN ceramics (a) $x=0$; (b) $x=0.01$; (c) $x=0.02$; (d) $x=0.03$

\subsection{BNT-ST- $x$ NN 陶瓷介电温谱图}

图 3 为极化后 BNT-ST- $x \mathrm{NN}(x=0.01 \sim 0.03)$ 陶瓷 的介电温谱图, 测试温区为 $25 \sim 400{ }^{\circ} \mathrm{C}$, 测试频率 范围 $100 \mathrm{~Hz} 1 \mathrm{MHz}$ 。从图中可以得知所有组分的陶 瓷都存在频率色散和介电峰宽化现象，且随着 NN
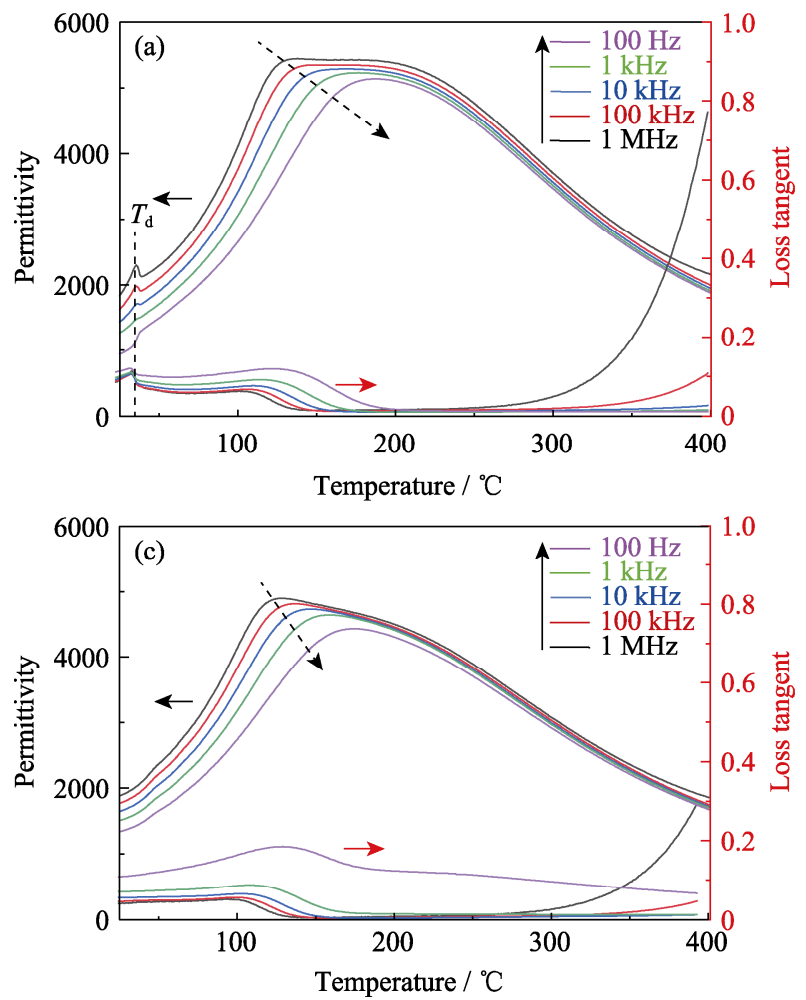

图 3 BNT-ST- $x$ NN 极化陶瓷的介电温谱图

Fig. 3 Dielectric temperature spectra of poled BNT-ST- $x$ NN ceramics

(a) $x=0$; (b) $x=0.01$; (c) $x=0.02$; (d) $x=0.03$

Colorful figures are available on website
增加，陶瓷的介电峰宽化逐渐增强，而相应介电常 数随之降低。BNT 基陶瓷中，宽化的介电峰通常涉 及三方与四方 PNRs 的局域热演化 ${ }^{[23]}$ 。显然, 所有 组分的陶瓷都为典型的弛豫铁电陶瓷，且掺杂 NN 能强化陶瓷的介电弛豫性, 介电常数的热稳定性明 显提升。值得注意的是，除 $x=0$ 陶瓷的宽化介电峰 外, 在 $30{ }^{\circ} \mathrm{C}$ 附近出现低温介电异常现象, 如图 3(a) 所示, 此处介电损耗出现峰值, 介电常数发生突变, 对应于高温遍历弛豫态到低温非遍历弛豫态的转变, 一般可将此温度记为 $T_{\mathrm{d}}$ 。显然, $x=0$ 陶瓷“非遍历弛 豫态”转变为“遍历弛豫态”发生在室温, 而其余组 分陶瓷 $(x>0)$ 在室温下主要呈现“遍历弛豫态”。

\subsection{BNT-ST $-x$ NN 陶瓷室温铁电电滞回线和电}

\section{流-电场曲线}

BNT 基陶瓷的弛豫相演变过程可反映在图 4 的 铁电电滞回线 $(P-E)$ 中, $x=0$ 时并非典型的正常铁电 $P-E$ 曲线, 而呈现束腰状, 且随着 $x$ 增大, $P-E$ 曲线 逐渐收缩, 剩余极化值 $P_{\mathrm{r}}$ 、矫顽场 $E_{\mathrm{c}}$ 以及最大极化 值 $P_{\max }$ 均呈下降趋势, 说明 $\mathrm{NN}$ 强化了陶瓷的遍历 弛豫态, 诱导更多铁电畴转变为可逆的 PNRs, 可在 电场撤除后回到初始状态，此结果与介电温谱图相 互印证。此外, 在 $x=0.03$ 时 $P_{\mathrm{r}}$ 与 $E_{\mathrm{c}}$ 都接近零, 表明室 温下该组分陶瓷的遍历弛豫态已占据绝对主导位置。
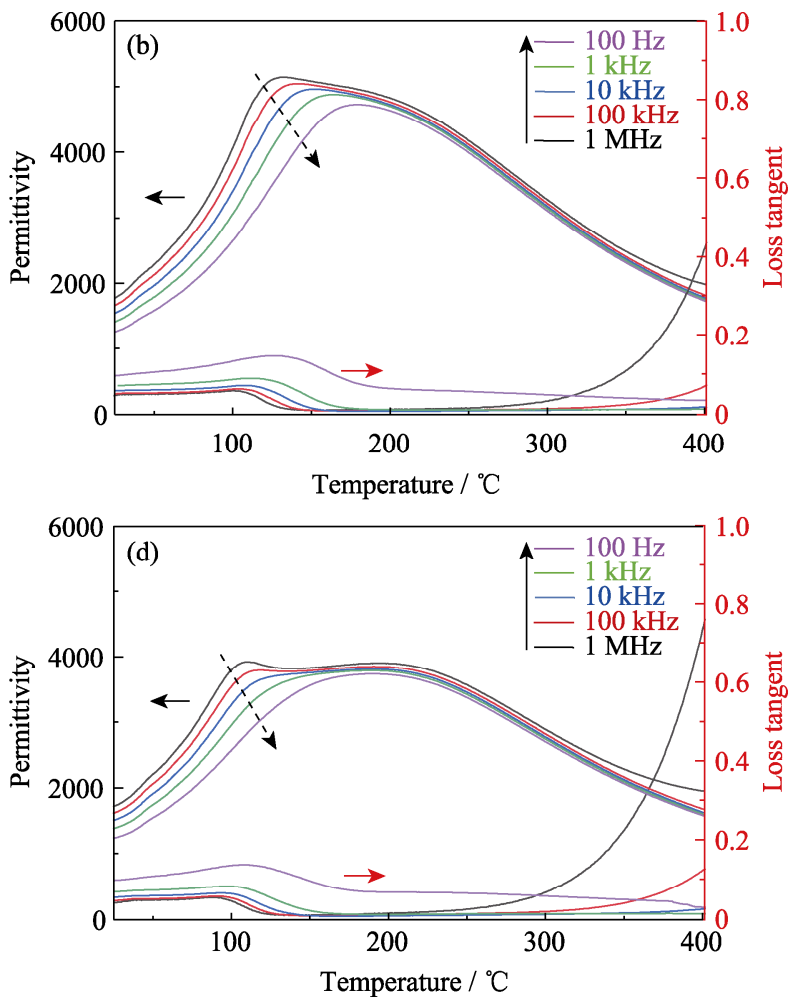

Temperature $/{ }^{\circ} \mathrm{C}$ 

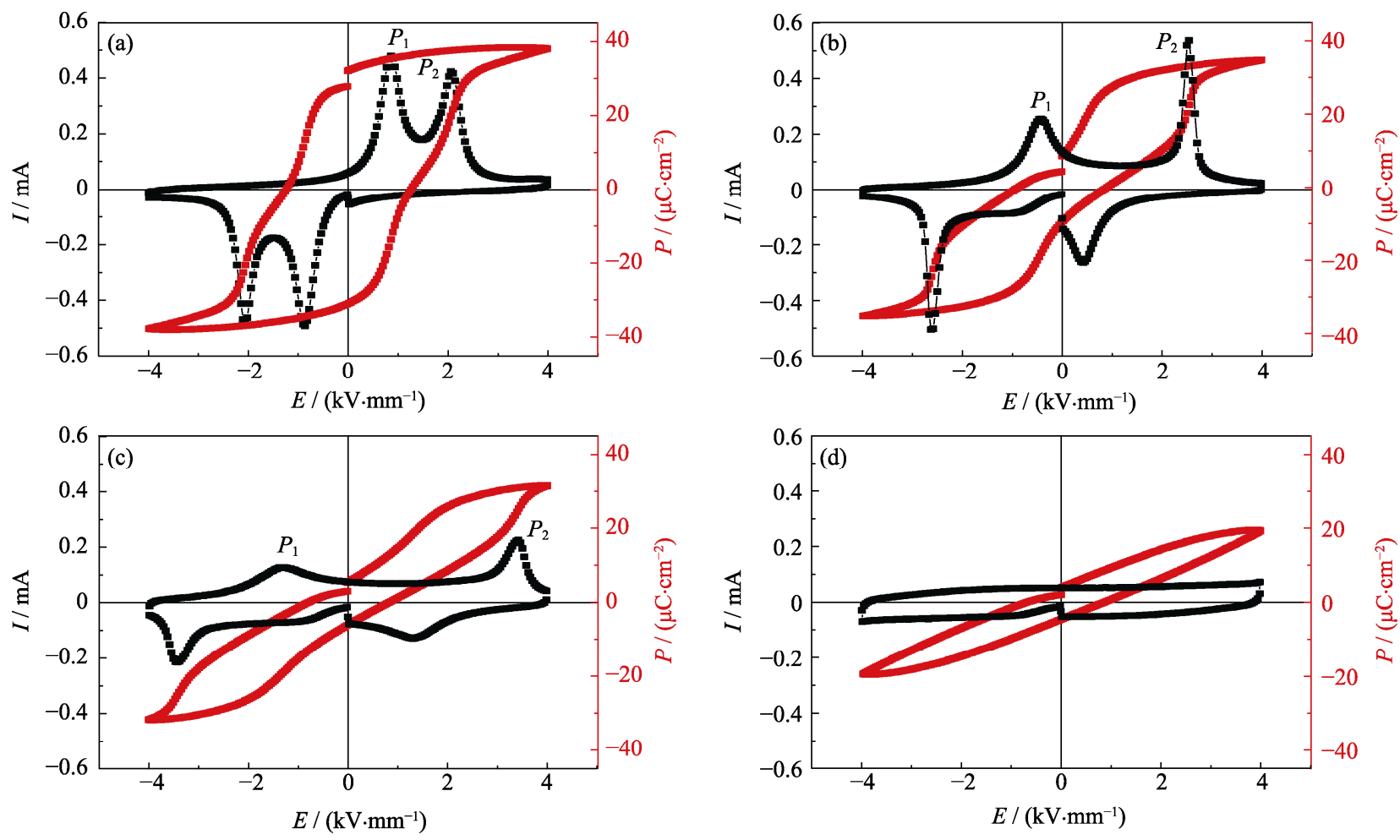

图 4 BNT-ST- $x$ NN 陶瓷室温铁电电滞回线和电流-电场曲线

Fig. 4 Room-temperature $P$-E loops and $I-E$ curves of BNT-ST- $x$ NN ceramics

(a) $x=0$; (b) $x=0.01$; (c) $x=0.02$; (d) $x=0.03$

Colorful figures are available on website

更精确的弛豫行为演变过程可在 $I-E$ 曲线上展 现，图 4(a)显示 $x=0$ 陶瓷的电流曲线在第一、三象 限都存在两个电流峰 $P_{1}$ 和 $P_{2}$, 这可归因于在外加电 场下 PNRs 分两步转变为长程有序的铁电畴分两步 转变为长程的铁电畴: 第一步为低电场下 PNRs 转 变为亚稳态微米尺度铁电畴, 此时宏观对称性不改 变; 第二步较高电场诱导亚稳态微米畴沿电场方向 翻转, 此时宏观上为铁电相 ${ }^{[24]}$ 。值得注意的是, 随 着 $\mathrm{NN}$ 量增大 $(x>0)$, 电流峰 $P_{1}$ 与 $P_{2}$ 分别由一、三 象限转变到四、二象限, 这表明此样品的部分铁电 畴在电场逐渐撤除后已无法维持, 出现退极化现象, 遍历弛豫态逐渐占据主导。图 4(d)中电流峰已经难 以观测, 剩余极化强度接近零, 说明 $x=0.03$ 时, $4 \mathrm{kV} / \mathrm{mm}$ 的电场已难以克服随机场诱导弛豫态到铁 电相的转变。

\subsection{BNT-ST- $x$ NN 陶瓷室温场致应变曲线}

图 5 可观察到 NN 对 BNT-ST 陶瓷的场致应变 性能影响。随着 NN 量增大, 场致应变 $(S-E)$ 曲线逐 渐由“蝴蝶状”变为“豆芽状”, 负向应变 $S_{\text {neg }}$ 值逐渐 减小, 在 $x=0.01$ 时已接近零。其次, 正向应变 $S_{\mathrm{pos}}$ 值在 $x=0.01$ 处最大, 在电场 $E=4 \mathrm{kV} / \mathrm{mm}$ 时, 场致应 变 $S_{\mathrm{pos}}=0.278 \%, d_{33}^{*}$ 可达 $695 \mathrm{pm} / \mathrm{V}$ 。与单纯的 BNT-ST 相比, 驱动电场更低但应变值却更高, 这证 明掺杂适量 $\mathrm{NN}$ 有利于降低驱动电场，同时保持良
好的场致应变。优异的场致应变性能可归于 $x=0.01$ 陶瓷在室温下处于 MPB(II), 此时电场诱导弛豫-铁 电相转变所需要克服的势垒降低, 且可逆的非 $180^{\circ}$ 铁电畴增加 ${ }^{[25]}$ 。此外, $x=0.02$ 时, 应变值也可达到 $0.249 \%$, 此组分场致应变值虽较 $x=0$ 陶瓷略微降低, 但是应变迟滞却明显从 $58 \%$ 下降至 $43 \%$ 。此现象 的主要原因在于, $x>0.01$ 时, 遍历弛豫态已占据主 导地位, 在同一电场下难以转变为铁电相, 从而电 畴翻转造成的迟滞降低。

\subsection{BNT-24ST- $x$ NN 陶瓷片在不同温度下的场}

\section{致应变图}

图 6 和图 7 分别为 BNT-ST-0.01NN 与 BNT-ST$0.02 \mathrm{NN}$ 陶瓷在 $25 \sim 100{ }^{\circ} \mathrm{C}$ 下的场致应变图。可以观 察到, 对这两种组分的陶瓷而言, 温度升高伴随着 迟滞降低, 可归因于温度升高使 PNRs 活性增加。

其次, $x=0.02$ 陶瓷的场致应变值随温度升高单调减 少, 而 $x=0.01$ 陶瓷的场致应变存在先升高再降低的 过程。图 6(b) 中可看到, $x=0.01$ 陶瓷在 $50{ }^{\circ} \mathrm{C}$ 下，应 变值为 $0.31 \%$, 高于室温值, 这主要源于掺杂 $\mathrm{NN}$ 与 升高温度对弛豫相的形成可造成叠加效应。当 $x=0.01$ 时, 不可逆的非 $180^{\circ}$ 铁电畴仍然少量残存, 在图 6(a)中反映为负向应变, 并非完全消失, 此时 适度升高温度导致 PNRs 增多, 可逆的非 $180^{\circ}$ 畴增 多，从而场致应变升高 ${ }^{[24]}$ 。此外，温度进一步升高 

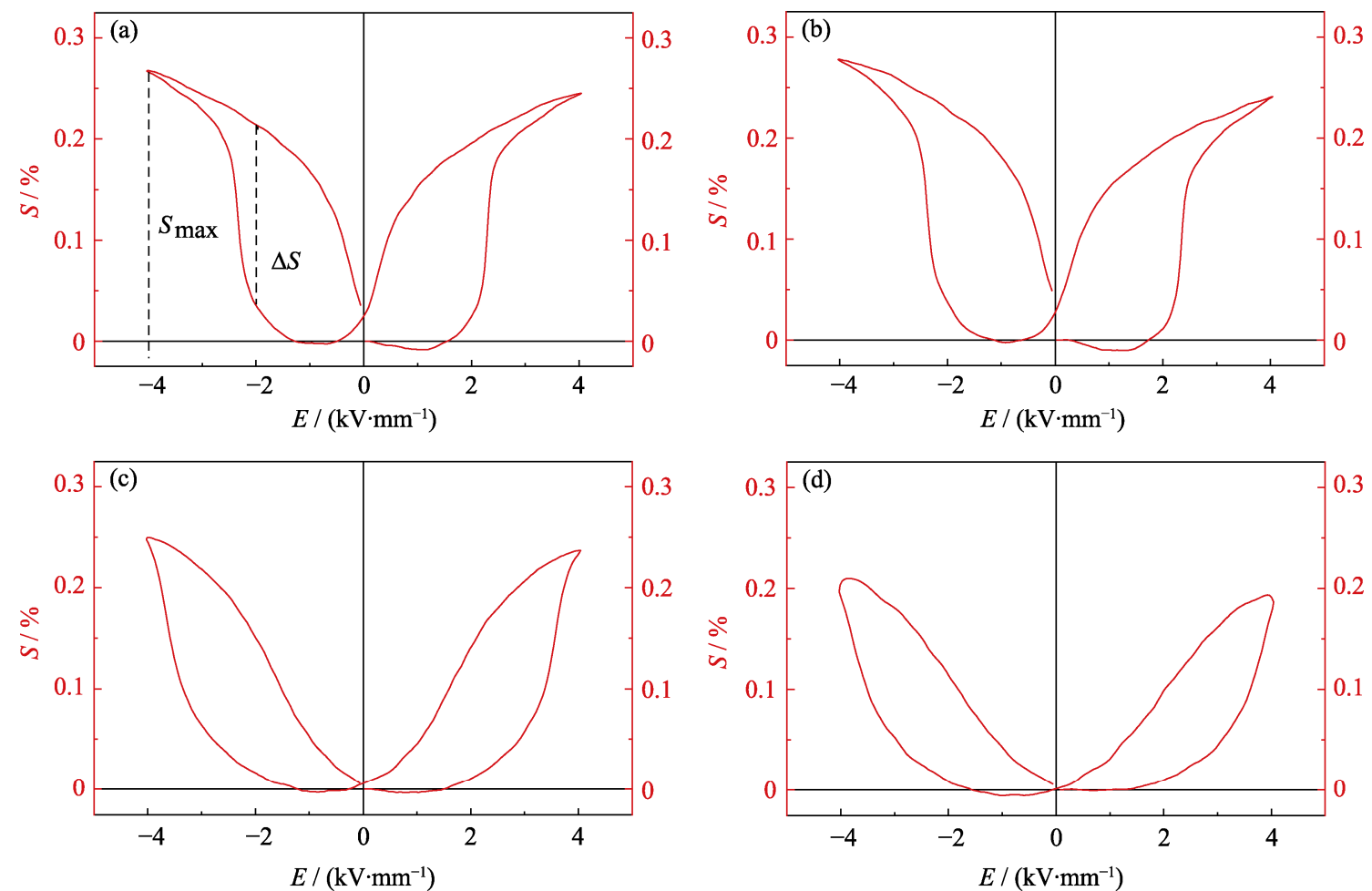

图 5 BNT-ST- $x$ NN 陶瓷室温场致应变图

Fig. 5 Field-induced strain curves of BNT-ST- $x$ NN ceramics at room temperature

(a) $x=0$; (b) $x=0.01$; (c) $x=0.02$; (d) $x=0.03$
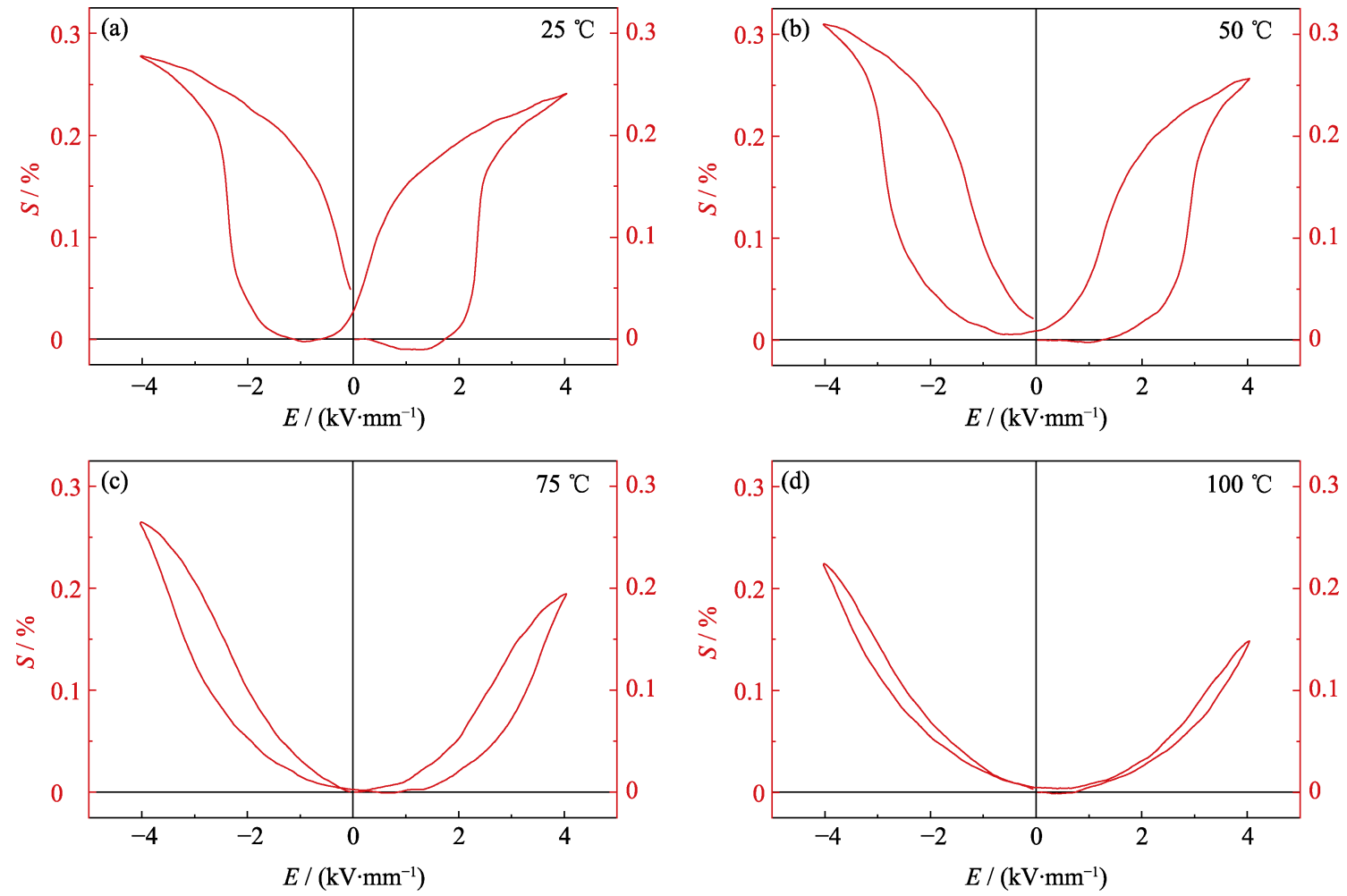

图 6 BNT-ST-0.01NN 陶瓷在不同温度下的场致应变图

Fig. 6 Field-induced strain curves of BNT-ST-0.01NN ceramics at different temperatures

(a) $25{ }^{\circ} \mathrm{C}$; (b) $50{ }^{\circ} \mathrm{C}$; (c) $75{ }^{\circ} \mathrm{C}$; (d) $100{ }^{\circ} \mathrm{C}$

使得 PNRs 活性更高, 从而电场诱导弛豫-铁电相转 变所需要克服的势垒也相应地升高，导致相同电场 下场致应变值下降 ${ }^{[26]}$ 。最后, $x=0.02$ 时, 场致应变随
NN 增加单调递减源自此时遍历弛豫态已占据主导 地位，温度继续升高致使所需驱动电场增加。从上 述讨论可得, 在较低驱动电场下, 可在 $x=0.01$ 处适 

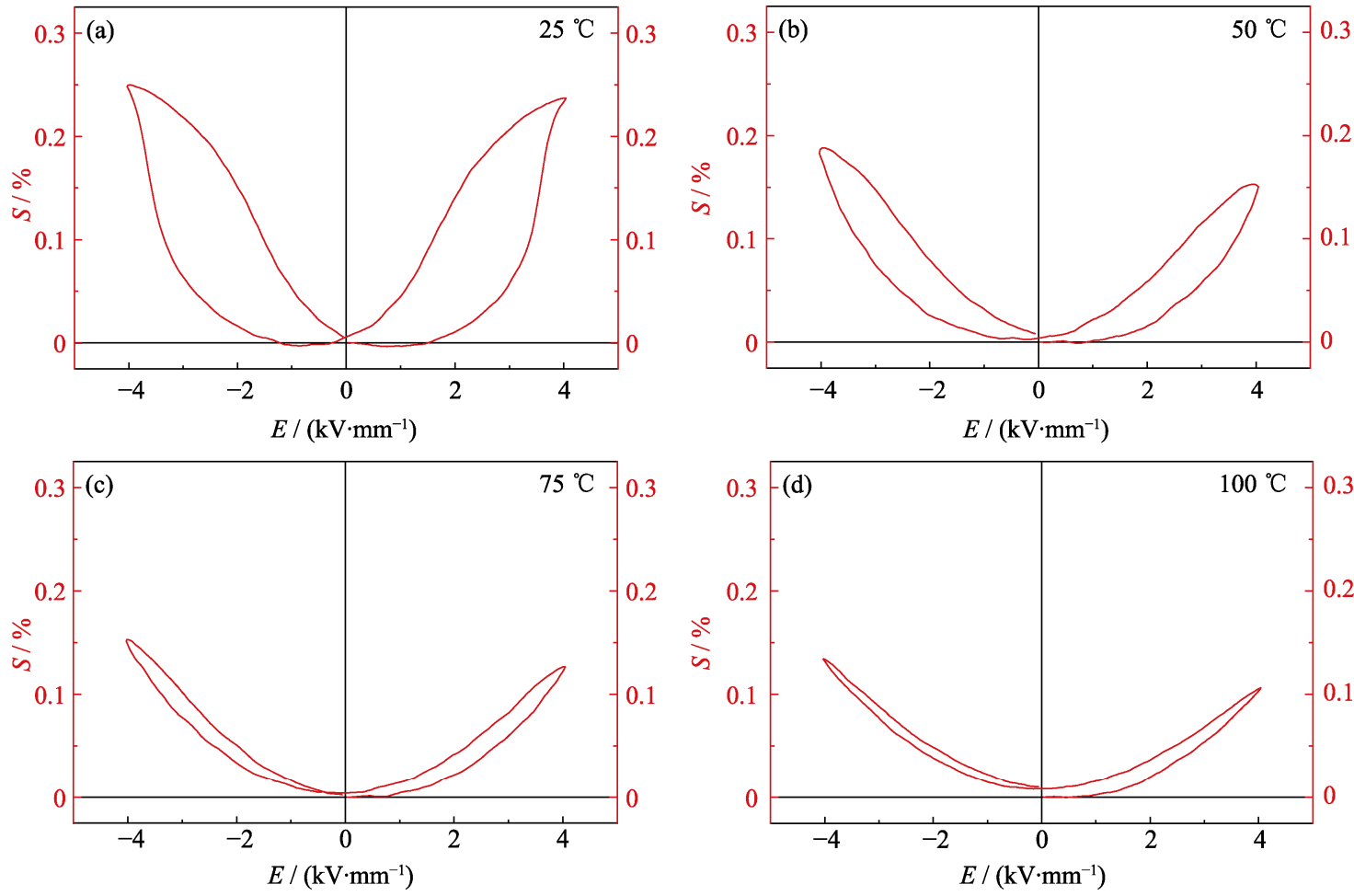

图 7 BNT-ST-0.02NN 陶瓷在不同温度下的场致应变图

Fig. 7 Field-induced strain curves of BNT-ST-0.02NN ceramics at different temperatures (a) $25{ }^{\circ} \mathrm{C}$; (b) $50{ }^{\circ} \mathrm{C}$; (c) $75{ }^{\circ} \mathrm{C}$; (d) $100{ }^{\circ} \mathrm{C}$

当升温或者进一步加入少量 $\mathrm{NN}(0.01<x<0.02)$ 来提 高应变。同时，在 $x=0.01 \sim 0.02$ 时，陶瓷场致应变的 温度稳定性良好。

\section{3 结论}

1) BNT-ST- $x \mathrm{NN}(x=0 \sim 0.03)$ 陶瓷均呈伪立方相, 且随着 $\mathrm{NN}$ 加入量增大, 遍历弛豫性逐渐增强, 铁电 畴转变为 PNRs, 使得 $P_{\mathrm{r}} 、 E_{\mathrm{c}}$ 以及负应变都逐渐下降。

2) $\mathrm{NN}$ 诱导陶瓷从非遍历弛豫态向遍历弛豫态 转变, 在 $x=0.01$ 时临近相界处, 此时 $T_{\mathrm{d}}$ 已低于室温, 在驱动电场 $E=4 \mathrm{kV} / \mathrm{mm}$ 下, 场致应变值 $S_{\mathrm{pos}}=0.278 \%$, $d_{33}^{*}$ 可达 $695 \mathrm{pm} / \mathrm{V}$, 场致应变过程发生铁电畴翻转, 因此应变迟滞较高 $58 \%$ 。

3) BNT-ST-0.01NN 陶瓷在温度为 $50{ }^{\circ} \mathrm{C}$ 左右应 变值 $S_{\mathrm{pos}}=0.31 \%, d_{33}^{*}$ 可达 $775 \mathrm{pm} / \mathrm{V}$ 。适当升温可以 提升 PNRs 的活性, 可逆的非 $180^{\circ}$ 畴增加。通过适当 调控温度也可提升应变值, 降低迟滞。

4) BNT-ST-0.02NN 在驱动电场 $E=4 \mathrm{kV} / \mathrm{mm}$ 下, 室温 $S_{\text {pos }}$ 为 $0.249 \%$, 与 BNT-ST-0.01NN 相比略微下 降，但迟滞却降低至 $43 \%$ 。

\section{参考文献:}

[1] 范鹏元. 大电致应变钛酸铋钠基无铅陶瓷的制备及性能研究.
武汉: 华中科技大学博士学位论文, 2018 .

[2] HAUN M J, FURMAN E. Modeling of the electrostrictive, dielectric, and piezoelectric properties of ceramic $\mathrm{PbTiO}_{3}$. IEEE Transactions on Ultrasonics Ferroelectrics \& Frequency Control, 1989, 36(4): 393-401.

[3] RÖDEL J, JO W, SEIFERT K T P, et al. Perspective on the development of lead-free piezoceramics. Journal of the American Ceramic Society, 2009, 92(6): 1153-1177.

[4] JAFFE B. Antiferroelectric ceramics with field-enforced transitions: a new nonlinear circuit element. Proceedings of the IRE, 1961, 49(8): 1264-1267.

[5] WU L, ZHANG J L, WANG C L, et al. Influence of compositional ratio $\mathrm{K} / \mathrm{Na}$ on physical properties in $\left(\mathrm{K}_{x} \mathrm{Na}_{1-x}\right) \mathrm{NbO}_{3}$ ceramics. Journal of Applied Physics, 2008, 103: 084116.

[6] LV X, WU J G, XIAO D Q, et al. Modifying temperature stability of $(\mathrm{K}, \mathrm{Na}) \mathrm{NbO}_{3}$ ceramics through phase boundary. Advanced Electronic Materials, 2018, 4(9): 1800205.

[7] CHAO C, ZHAO X, WANG Y, et al. Giant strain and electricfield-induced phase transition in lead-free $\left(\mathrm{Na}_{0.5} \mathrm{Bi}_{0.5}\right) \mathrm{TiO}_{3}-\mathrm{BaTiO}_{3}$ $\left(\mathrm{K}_{0.5} \mathrm{Na}_{0.5}\right) \mathrm{NbO}_{3}$ single crystal. Applied Physics Letters, 2016, 108(2): 022903.

[8] SAITO Y, TAKAO H, TANI T, et al. Lead-free piezoceramics. Nature, 2004, 432(7013): 84-87.

[9] 黄宇. 钣酸铋钠基无铅陶瓷的储能性能研究. 武汉: 武汉理工 大学硕士学位论文, 2019.

[10] ACOSTA M, NOVAK N, ROJAS V, et al. $\mathrm{BaTiO}_{3}$-based piezoelectrics: fundamentals, current status, and perspectives. Applied Physics Reviews, 2017, 4(4): 041305.

[11] ZHAO C L, WU B, THONG H C, et al. Improved temperature stability and high piezoelectricity in lead-free barium titanatebased ceramics. Journal of the European Ceramic Society, 2018, 38(16): 5411-5419.

[12] TAN Y, ZHANG J, WANG C, et al. Enhancement of electric 
field-induced strain in $\mathrm{BaTiO}_{3}$ ceramics through grain size optimization. Physica Status Solidi, 2015, 212(2): 433-438.

[13] ZHAO Z H, LI X L, DAI Y J, et al. Texture development in $\mathrm{Ba}_{0.85} \mathrm{Ca}_{0.15} \mathrm{Ti}_{0.90} \mathrm{Zr}_{0.10} \mathrm{O}_{3}$ lead-free ceramics prepared by reactive template grain growth with different $\mathrm{Ba}$ and $\mathrm{Ca}$ sources. Ceramics International, 2016, 42(16): 18756-18763.

[14] ZHANG S T, KOUNGA A B, AULBACH E, et al. Giant strain in lead-free piezoceramics $\mathrm{Bi}_{0.5} \mathrm{Na}_{0.5} \mathrm{TiO}_{3}-\mathrm{BaTiO}_{3}-\mathrm{K}_{0.5} \mathrm{Na}_{0.5} \mathrm{NbO}_{3}$ system. Applied Physics Letters, 2007, 91(11): 112906.

[15] ZHENG T, WU J, XIAO D, et al. Recent development in lead-free perovskite piezoelectric bulk materials. Progress in Materials Science, 2018, 98(1): 552-624.

[16] LI F, ZHANG S, YANG $\mathrm{T}$, et al. The origin of ultrahigh piezoelectricity in relax-ferroelectric solid solution crystals. Nature Communications, 2016, 7(1): 13807.

[17] HAO J, SHEN B, ZHAI J, et al. Switching of morphotropic phase boundary and large strain response in lead-free ternary $\left(\mathrm{Bi}_{0.5} \mathrm{Na}_{0.5}\right) \mathrm{TiO}_{3}-\left(\mathrm{K}_{0.5} \mathrm{Bi}_{0.5}\right) \mathrm{TiO}_{3}-\left(\mathrm{K}_{0.5} \mathrm{Na}_{0.5}\right) \mathrm{NbO}_{3}$ system. Journal of Applied Physics, 2013, 113(11): 114106.

[18] CHEN J, WANG Y, ZHANG Y, et al. Giant electric field-induced strain at room temperature in $\mathrm{LiNbO}_{3}$-doped $0.94\left(\mathrm{Bi}_{0.5} \mathrm{Na}_{0.5}\right) \mathrm{TiO}_{3}$ 0.06BaTiO ${ }_{3}$. Journal of the European Ceramic Society, 2017, 37(6): 2365-2371.

[19] HIRUMA Y, IMAI Y, WATANABE Y, et al. Large electrostrain near the phase transition temperature of $\left(\mathrm{Bi}_{0.5} \mathrm{Na}_{0.5}\right) \mathrm{TiO}_{3}-\mathrm{SrTiO}_{3}$ ferroelectric ceramics. Applied Physics Letters, 2008, 92(26): 262904.

[20] NGUYEN H, DUONG T A, ERKINOV F, et al. Large electric field-induced strain response under a low electric field in lead-free $\mathrm{Bi}_{1 / 2} \mathrm{Na}_{1 / 2} \mathrm{TiO}_{3}-\mathrm{SrTiO}_{3}-\mathrm{BiAlO}_{3}$ ternary piezoelectric ceramics. Journal of Electronic Materials, 2020, 49(11): 6677-6685.

[21] TONG X Y, LI H L, ZHOU J J, et al. Giant electrostrain under low driving field in $\mathrm{Bi}_{1 / 2} \mathrm{Na}_{1 / 2} \mathrm{TiO}_{3}-\mathrm{SrTiO}_{3}$ ceramics for actuator applications. Ceramics International, 2016, 42(14): 16153-16159.

[22] HIRUMA Y, NAGATA H, TAKENAKA T. Phase diagrams and electrical properties of $\left(\mathrm{Bi}_{1 / 2} \mathrm{Na}_{1 / 2}\right) \mathrm{TiO}_{3}$-based solid solutions. Journal of Applied Physics, 2008, 104(12): 124106.

[23] ZHU Y, ZHANG Y, XIE B, et al. Large electric field-induced strain in $\mathrm{AgNbO}_{3}$-modified $0.76 \mathrm{Bi}_{0.5} \mathrm{Na}_{0.5} \mathrm{TiO}_{3}-0.24 \mathrm{SrTiO}_{3}$ lead-free piezoceramic. Ceramics International, 2018, 44(7): 7851-7857.

[24] HE H, LU X, LI M, et al. Thermal and compositional driven relaxor ferroelectric behaviours of lead-free $\mathrm{Bi}_{0.5} \mathrm{Na}_{0.5} \mathrm{TiO}_{3}-\mathrm{SrTiO}_{3}$ ceramics. Journal of Materials Chemistry C, 2020, 8(7): 2411-2418.

[25] BAI W, CHEN D, ZHENG P, et al. $\mathrm{NaNbO}_{3}$ templates-induced phase evolution and enhancement of electromechanical properties in grain oriented lead-free BNT-based piezoelectric materials. Journal of the European Ceramic Society, 2017, 37(7): 2591-2604

[26] MAURYA D, ZHOU Y, WANG Y, et al. Giant strain with ultra-low hysteresis and high temperature stability in grain oriented lead-free $\mathrm{K}_{0.5} \mathrm{Bi}_{0.5} \mathrm{TiO}_{3}-\mathrm{BaTiO}_{3}-\mathrm{Na}_{0.5} \mathrm{Bi}_{0.5} \mathrm{TiO}_{3}$ piezoelectric materials. Scientific Reports, 2015, 5(1): 8595. 